\title{
Identification and simulation of the impact characteristics of the viscous mount
}

\author{
H. Andou ${ }^{1}$, T. Koizumi ${ }^{2}$ \& N. Tsujiuchi ${ }^{2}$ \\ ${ }^{1}$ Test \& Validation Department, Shin Caterpillar Mitsubishi Ltd., Japan \\ ${ }^{2}$ Department of Mechanical Engineering, Doshisha University, Japan
}

\begin{abstract}
Hydraulic excavators have many different front attachments, and they are operated in various manners. Accordingly, stationary excitation and excessive impact loads are applied to the operator cabin. In recent years, a variety of mounts, including high viscous oil mounts, have been used to reduce the cabin vibration. Many papers have shown the stationary characteristics of the viscous mount has frequency- and amplitude-dependency, but there are few papers to characterize the impact behaviour of the viscous mount. The objective of this paper is to understand the impact behaviour of viscous mounts, which corresponds to impact loads for the actual machine. First, the reaction force and the response acceleration of the viscous mounts installed on the vehicle are measured during a critical drop impact test. Next, the impact test of the mount is performed to identify its impact characteristics. The test is performed with a shock load equivalent to the load applied during the drop impact test for the actual vehicle. The equivalent mass of the cabin is also applied to the mount during this shock load test. The characteristics are estimated from the measured data of the mount force and displacement at the initial impact by the least square method. Then, the response acceleration on the mount is calculated by the estimated characteristics using the impact load measured on the drop impact test for the actual vehicle. Finally, it is found that the identified characteristics are appropriate to correlate the calculated acceleration with the measured acceleration on the vehicle.
\end{abstract}

\section{Introduction}

Hydraulic excavator is a general-purpose construction machine. Therefore, a large variety of front attachments can be installed, for example, hammer, crusher, 
etc. instead of the bucket on a Hydraulic Excavator, Fig.1. Various vibrations are transmitted to the cabin through the front linkage, under carriage, and swing frame structures from the ground due to the front attachments. It is important to understand not only the stationary but also the impact characteristics for the development of the new cabin's mount. However, there are few papers to report the impact characteristics of the viscous mount to reduce the cabin's vibration.

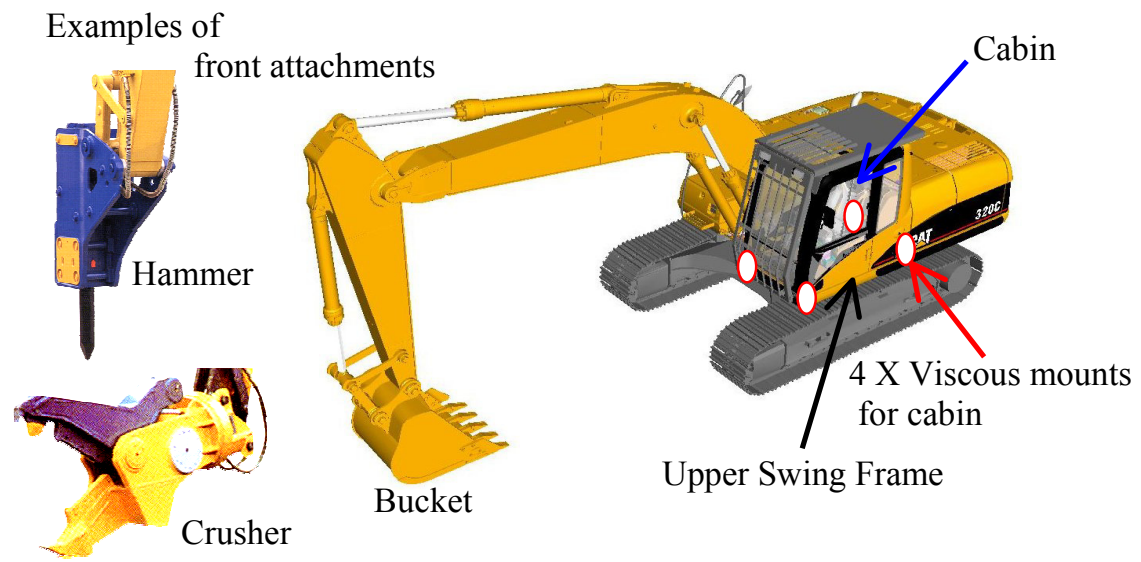

Figure 1: Hydraulic excavator.

Many cabin's mounts continue to be developed in order to improve a further ride comfort. But there are some concerns to abruptly install a prototype mount in a real machine. It is feared that the cabin will interfere with the components around it and the interior parts will break when the excessive impact load is applied.

It is necessary that the impact characteristics are understood and the response behaviour is accurately simulated before the mount is installed on the actual vehicle to prevent harm to the structure and operator. In this paper, the impact characteristics of the viscous mount are clarified and the simulation procedure to accurately simulate the reaction behaviour is reported when the impact force equivalent to the actual machine operation is applied to the mount Fig.2. At first, the vehicle drop impact test is performed to measure the mount reaction force and the response acceleration on the mount. Next, the impact characteristics of the mount itself are identified by the measured displacement from the iron ball falling test. Then, the response acceleration on the mount is simulated using the identified characteristics when the measured force in the actual vehicle is applied.

Finally, it is verified that the identified impact characteristics are appropriate by comparing the simulated result with the measured acceleration in the vehicle drop impact test. 


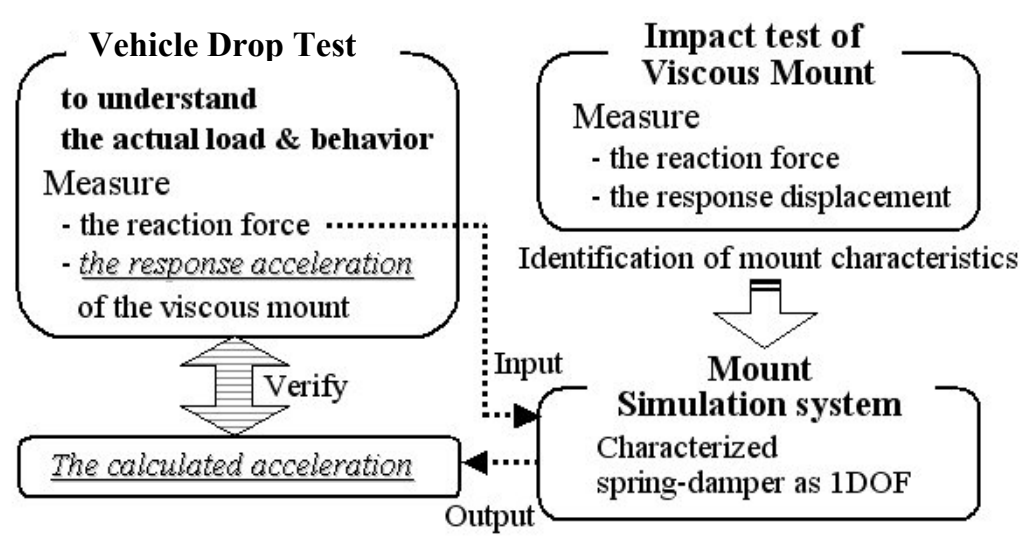

Figure 2: $\quad$ Outline of this study.

\section{Drop impact test for the actual machine}

The impact absorption performance of the viscous mount is one of the important verification items in the development and design stage of hydraulic excavators since the impact load frequently occurs in operation. As the impact often provides an extremely large deformation to the mounts, it increases the probability of interference between the cabin and the other components such as swing frame and side cover etc. Allowing a large stroke of the mount is difficult in actual design because of machine layout constraints. It is necessary to estimate the peak (acceleration or displacement) of the mount as accurately as possible when the impact force is applied to the mount.

When the excessive lifting load is applied at the tip of the front attachment for a vehicle, the rear portion of the vehicle lifts up as the fulcrum on the forward of the crawler. After the load is released, the vehicle falls down on the ground and the shock is applied to the cabin supported by the mount. At first, the impact force is measured for the actual vehicle before the impact characteristics of the mount itself are understood. Assuming the drop impact frequently occurs, the drop impact test shown in Fig. 3 is performed at the maximum angle observed for the actual operations. The bucket is hung on the hook installed on the concrete wall in order to reproduce the posture when the rear portion of the vehicle lifts up and the vehicle is dropped by removing the bucket from the hook. The time history of the reaction force and the response acceleration at the front-left mount are measured using the load cell (Piezotronics; Ring-type Load cell, 204C, Max $180 \mathrm{kN}$ ) and the two accelerometers (Piezotronics; ICP Accelerometer, 356A32, Measurement Range $\pm 50 \mathrm{G}$ peak). The mounting locations of the sensors are shown in Fig.4.

Fig. 5 shows the measured data of the force and accelerations, respectively. The acceleration result is calculated as the relative acceleration of the mount from the measured accelerations (a)-(b), referring to Fig 4. 


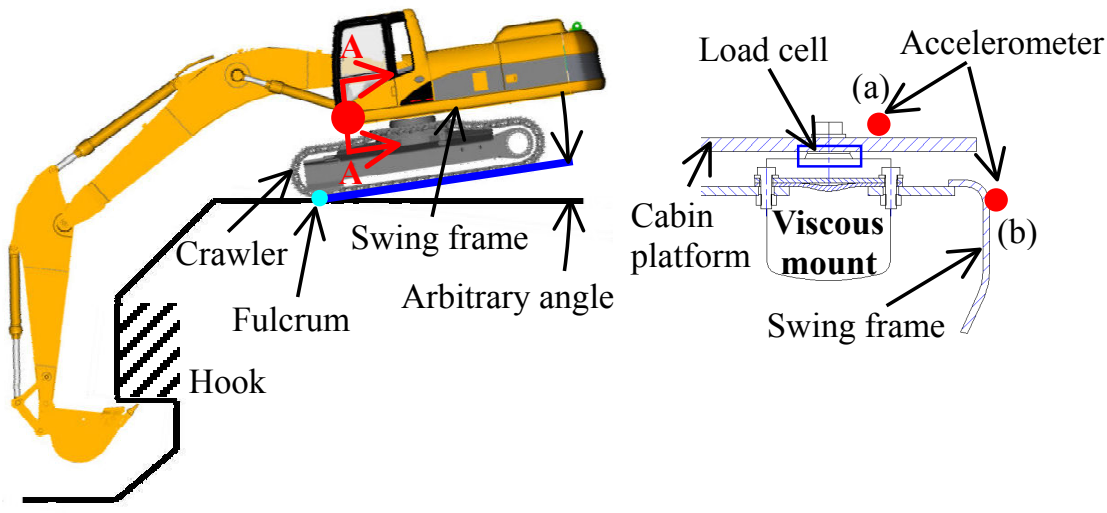

Figure 3: Drop impact test. Figure 4: Viscous mount location (Section AA).

The maximum reaction force is $7393 \mathrm{~N}$ and the maximum response acceleration is $13.4 \mathrm{~m} / \mathrm{s}^{2}$. The first impulse (sum of products at force and time) is 138.2[N-s].
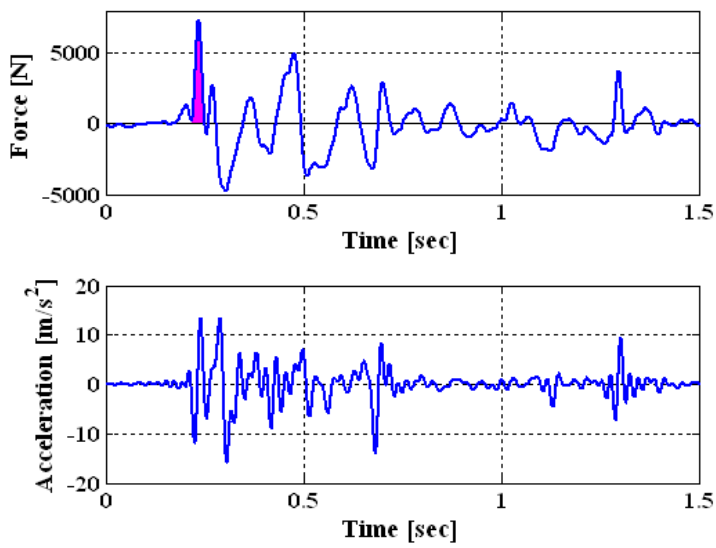

Figure 5: Vehicle measurements.

\section{Impact test of the viscous mount}

It is difficult to apply the actual impact load measured on the actual vehicle to identify the impact characteristics of the viscous mount. The actual cabin structure is assumed as a concentrated mass and then an iron ball drop test is performed (Fig.6). To simplify the test equipments and to lighten the weight of 
the iron ball, the concentrated mass is supported by three mounts, though the four mounts support the cabin on the vehicle. The static load applied on one mount is same as the actual supported cabin condition. In addition, the centre of the top surface for the equivalent mass is designed above of the centre of gravity as the impact force is uniformly loaded for each mount.

The change of the impact characteristics with regard to the impulse is investigated by executing the drop test under varying conditions in which the weight and height of the iron ball are changed. Fig.7 shows the measured data of the reaction force and the response displacement of the mount as an example of an equivalent impulse of the actual vehicle test. The equivalent condition is an iron ball weight of $38 \mathrm{~kg}$ and the height of $2 \mathrm{~m}$. The over damping characteristics of the viscous mount used for the hydraulic excavator is observed from the response displacement.

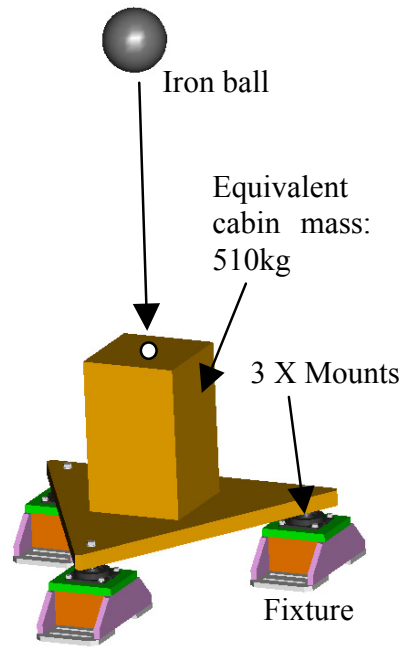

Figure 6: Mount impact test. Figure 7: Measured response of mount.

\section{Identification of the impact characteristics}

In order to understand the impact characteristics from the measured data of one mount for the above-mentioned mount impact test, we consider them as a springdamper system.

The equation of motion is represented by Equation (1), when an impulse force acts on a single-degree-of-freedom system.

$$
m \ddot{x}(t)+c \dot{x}(t)+K_{d} x(t)=\hat{I} \delta(t)
$$

where as $\hat{I}$; impulse, $\delta(t)$ : delta function 
The silicone oil with high viscosity is enclosed in the viscous mount in this study. It is known that the mount has the characteristic of overdamping from the frequency response obtained by test and analysis result [7]. Letting the initial conditions be $x(0)=\dot{x}(0)=0$, Equation (2) is obtained after solving Equation (1) by Laplace transform.

$$
X(s)=\frac{\hat{I}}{m} \cdot \frac{1}{\left(s+\zeta \omega_{n}\right)^{2}-\left(\zeta^{2}-1\right) \omega_{n}^{2}}
$$

And the Equation (2) is transferred to Equation (3) by inverse Laplace transform.

$$
\begin{gathered}
x(t)=\frac{\hat{I}}{2 \omega_{n} m \sqrt{\zeta^{2}-1}} \cdot\left[\exp \left\{-\omega_{n}\left(\zeta-\sqrt{\zeta^{2}-1}\right) t\right\}-\exp \left\{-\omega_{n}\left(\zeta+\sqrt{\zeta^{2}-1}\right) t\right\}\right] \\
\omega_{n}=\sqrt{K_{d} / m}, \varsigma=c / 2 \sqrt{m K_{d}}
\end{gathered}
$$

The equation of the displacement in the case of overdamping is derived. Equation (3) is valid in the case of a damping ratio less than one $(\zeta<1)$.

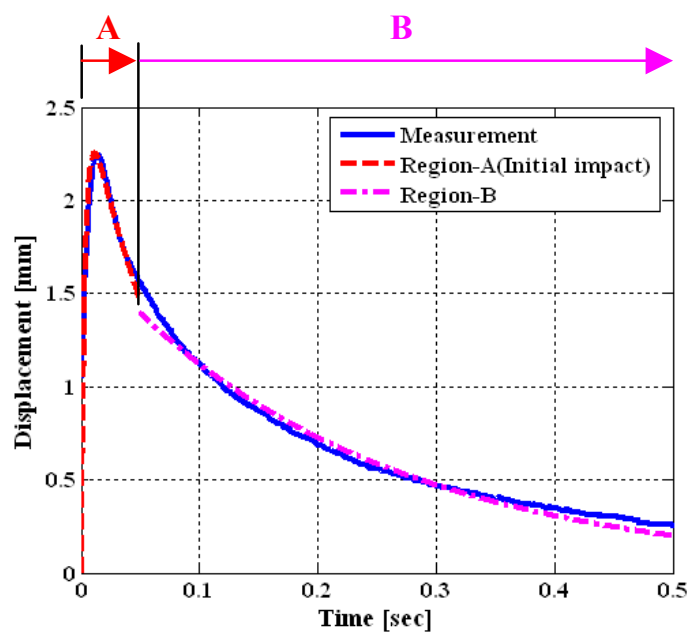

Figure 8: $\quad$ Identification of impact characteristics.

The solid line in Fig.8 shows an example of the time histories of the response mount displacement obtained by the impact test. When the impact force is applied on the mount, the displacement reaches a maximum, and then the rebound begins. The mount displacement behaves differently in two regions, the first region is the downward stroke before the peak displacement and the other is the rebound region. The impulse response behaviour is different before and after $0.05 \mathrm{~s}$ [Region A (under damping) is quite different from the stationary one. Region B (over damping) is similar to a stationary one.]. It is shown that the characteristics in the initial impact stage are different from the characteristics after the initial stage. The reaction of squeezing the silicone oil is predominant 
because the high viscosity oil doesn't instantaneously flow in the first stage of the impact causing the viscous damping of the mount to lag the impact. The identified results allow the calculation of the peak displacement, its timing, and overall damped displacement. Therefore, the damping properties: the natural angular frequency $\omega_{n}$ and the damping ratio $\zeta$, are separately identified in the region $\mathrm{A}$ and $\mathrm{B}$. The Gauss-Newton method, a nonlinear least-squares approximation, is used as shown in Equation (3) based on the mount displacement at the impact test. At first, a pair of $\omega_{n}$ and $\zeta$, and the dynamic stiffness $\mathrm{K}_{\mathrm{d}}$, the loss stiffness $\mathrm{C} \omega$ and the impulse $\hat{I}$ are identified in region A. $\hat{I}$ is included in the identified parameters due to a concern whether the impact force might be accurately measured in the very fast phenomenon. The dynamic stiffness and the loss stiffness are identified in the region B using the obtained $\hat{I}$.

The previous characteristics of the viscous mount are more important than the following characteristics with regard to the estimation of the maximum displacement of the overall cabin structure. The results of the identified characteristics in the region A for several test cases are plotted in Fig.9 and Fig. 10.

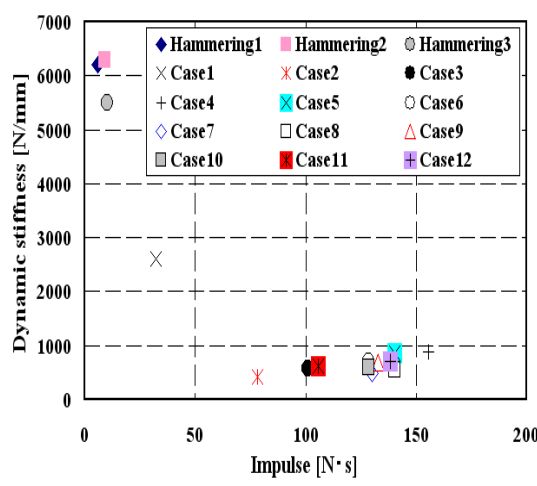

Figure 9: Identified dynamic stiffness

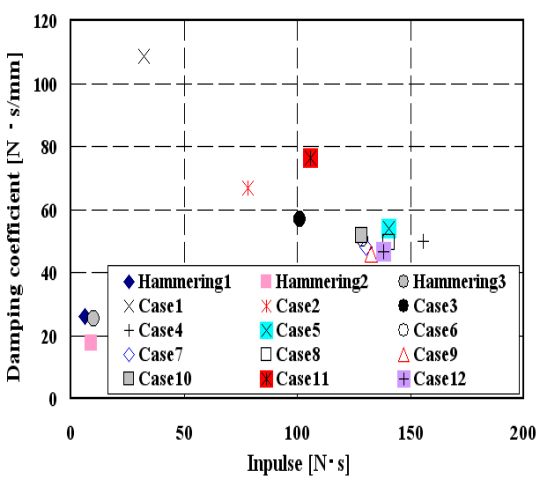

Figure 10: Identified damping coefficient

As a reference, the results in the case of a hammering test are plotted. The results of the iron ball drop test are entirely different from the results of hammering, but the results of the impulse range from 100 to $105 \mathrm{~N}$-s. Both the dynamic stiffness and the damping coefficients have a significant dependency corresponding to the impact force. Accordingly the identified characteristics determined by the impact force test of the actual vehicle should be used; when impact simulation of the cabin system mounts are performed. 


\section{Simulation and validation of the mount response}

This section describes a procedure to calculate the response acceleration of the mount with the objective to estimate the overall behaviour of the cabin's vibration. The viscous mount is assumed to be a one degree-of-freedom (spring-damper) system with viscous damping. The measured force on the actual vehicle test is substituted to the right side of Equation (1).

$$
m \ddot{x}(t)+c \dot{x}(t)+K_{d} x(t)=f(t)
$$

where as, $f(t)$ is the history of the applied force to the mount.

Using the impact characteristics identified previously and the measured history of the reaction force of the viscous mount for the drop impact test on the actual vehicle, the response acceleration is calculated. The calculated acceleration is compared with the measured acceleration for the vehicle test to verify the proposed method is valid.

The response acceleration is calculated by the direct time integration method. Equation (4) is reformed by introducing the new variable, $\mathrm{u}$.

$$
M=\left[\begin{array}{cc}
I & 0 \\
0 & m
\end{array}\right], L=\left[\begin{array}{cc}
0 & -I \\
K_{d} & c
\end{array}\right], F=\left\{\begin{array}{l}
0 \\
f
\end{array}\right\}, u=\left\{\begin{array}{l}
\dot{x} \\
x
\end{array}\right\}, \dot{u}=\left\{\begin{array}{l}
\ddot{x} \\
\dot{x}
\end{array}\right\}
$$

One of the best known methods for solving this equation is the fourth order Runge-Kutta method [8-10].

$$
u_{n+1}=u_{n}+\frac{p_{1}+2 p_{2}+2 p_{3}+p_{4}}{6}
$$

where

$$
p_{1}=\Delta t \cdot F\left(u_{n}\right), p_{2}=\Delta t \cdot F\left(u_{n}+p_{1} / 2\right), p_{3}=\Delta t \cdot F\left(u_{n}+p_{2} / 2\right), p_{4}=\Delta t \cdot F\left(u_{n}+p_{3}\right)
$$

The unknown variables $x_{n+1}$ and $\dot{x}_{n+1}$ are calculated from the already-known variables $x_{n}, \dot{x}_{n}$ and $\ddot{x}_{n}$. And the response acceleration can be obtained by Equation (8).

$$
\ddot{x}_{n+1}=m^{-1}\left(f-c \dot{x}_{n+1}-K_{d} x_{n+1}\right)
$$

The simulated and measured acceleration are plotted in Fig.11.

The first peak of the simulated acceleration has very good correlation with the measured data. The calculated peak acceleration has an error of $8.3 \%$ and $0.4 \%$ during the downward and upward motion, respectively.

Understanding the mount behaviour during the most severe condition for the drop impact is useful. It is critical for the first peak value to be correctly simulated to support the layout design of the vehicle.

\section{Conclusions}

- A method to identify the impact characteristics of the viscous mount used for a hydraulic excavator is suggested. 
- The impact characteristics are identified by the iron ball drop test and it is found that the impact characteristics vary significantly between the first impact region and the second region. In addition, the impact characteristics are different from the stationary characteristics.

- The response acceleration of the viscous mount simulated using the identified characteristics and the force measured on the drop impact test for the actual vehicle correlates well with the acceleration measured on the vehicle. It is found that the identified characteristics are appropriate and the computational procedure to calculate the impact response of the viscous mount described in this paper is effective.

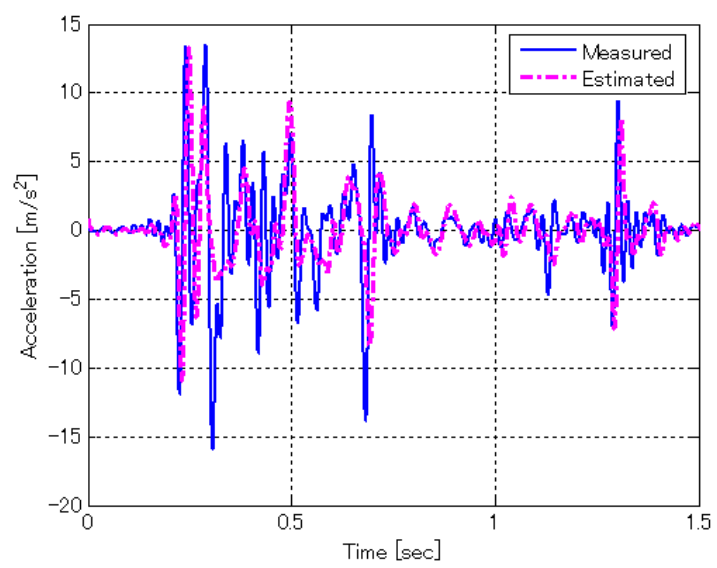

Figure 11: Response acceleration at impact.

\section{References}

[1] Hamazaki Y., Yonezawa S., Inoue Y., Study of Dynamic Behavior of Highly Viscous Fluid Mount, Trans. Jpn. Soc. Mech. Eng., Vol.95, No.1077, C (1996), p.907-912.

[2] Koizumi, T., Tsujiuchi, N., Shibayama, T., A Study of the Dynamic Characteristics of Hydraulic Engine Mount, Trans. Jpn. Soc. Mech. Eng., Vol.68, No.668, C (2002), p.1088-1095.

[3] Sugino, M., Okuzumi, H., Abe, E., Analysis and Application of Hydraulic Engine Mount, Nissan Technical Report, p.9-18 (1985).

[4] Song He, Rajendra Singh, Prediction of High Frequency Response Characteristics of Hydraulic Mounts, SAE Noise \& Vibration (2005).

[5] Song He, Rajendra Singh, Improved Estimation of Linear and Nonlinear Hydraulic Mount Models for Transient Responses, SAE Noise \& Vibration (2005).

[6] Li-Rong Wang, Ichiro Hagiwara, Jia-Cai Wang, Zhen-Hua Lu, Parameter Estimations for Characteristic Simulation Model of Hydraulically 
Damped Rubber Mount by Finite Element Method, SAE Noise \& Vibration (2005).

[7] H. Andou, T. Koizumi, N. Tsujiuchi, T. Shibayama, Numerical Model of Viscous Mount for Hydraulic Excavator, IMAC (2005).

[8] U.M. Ascher, R.M.M. Mattheij and R.D. Russell, Numerical Solution of Boundary Value Problems for Ordinary Differential Equations, Prentice Hall (1988).

[9] Howard B. Wilson, Louis H. Turcotte, David Halpern, Advanced Mathematics and Mechanics Applications Using MATLAB, Chapman \& Hall/CRC, p.261-262 (2003).

[10] C. W. Gear. Numerical Initial Value Problems in Ordinary Differential Equations. Prentice-Hall (1971). 